\title{
Validity of physical activity monitors during daily life in patients with COPD
}

\author{
Roberto A. Rabinovich ${ }^{1,8}$, Zafeiris Louvaris 2,8, Yogini Raste ${ }^{3}$, Daniel Langer ${ }^{4}$, \\ Hans Van Remoortel ${ }^{4}$, Santiago Giavedoni ${ }^{1}$, Chris Burtin ${ }^{4}$, Eloisa M.G. Regueiro ${ }^{5}$, \\ loannis Vogiatzis ${ }^{2}$, Nicholas S. Hopkinson ${ }^{3}$, Michael I. Polkey ${ }^{3}$, \\ Frederick J. Wilson ${ }^{6}$, William MacNee ${ }^{1}$, Klaas R. Westerterp ${ }^{7}$ and \\ Thierry Troosters ${ }^{4}$ on behalf of the PROactive consortium
}

Affiliations: 'ELEGI Colt Laboratory, Centre for Inflammation Research, The Queen's Medical Research Institute, University of Edinburgh, Edinburgh, ${ }^{3} \mathrm{NIHR}$ Respiratory Biomedical Research Unit of the Royal Brompton and Harefield NHS Foundation Trust and National Heart and Lung Institute, Imperial College London, London, and ${ }^{6}$ Precision Medicine, Pfizer Worldwide Research and Development, Sandwich, UK. ${ }^{2}$ Dept of Critical Care Medicine, Pulmonary Rehabilitation Centre, Evangelismos Hospital, M. Simou and G.P. Livanos Laboratories, National and Kapodistrian University of Athens, Thorax Foundation, Athens, Greece. ${ }^{4}$ Dept of Rehabilitation Sciences, Faculty of Kinesiology and Rehabilitation Sciences, Katholieke Universiteit Leuven, Leuven, Belgium. ${ }^{5}$ Laboratory of Respiratory Physiotherapy, Universidade Federal de São Carlos (UFSCar), São Carlos, Brazil. ${ }^{7}$ Dept of Human Biology, Maastricht University, Maastricht, The Netherlands. ${ }^{8}$ Both authors contributed equally.

Correspondence: R.A. Rabinovich, ELEGI Colt Laboratory, Centre for Inflammation Research, The Queen's Medical Research Institute, University of Edinburgh, 47 Little France Crescent, Edinburgh, EH16 4TJ, UK. E-mail: roberto.rabinovichवed.ac.uk

ABSTRACT Symptoms during physical activity and physical inactivity are hallmarks of chronic obstructive pulmonary disease (COPD). Our aim was to evaluate the validity and usability of six activity monitors in patients with COPD against the doubly labelled water (DLW) indirect calorimetry method.

80 COPD patients (mean \pm SD age $68 \pm 6$ years and forced expiratory volume in $1 \mathrm{~s} 57 \pm 19 \%$ predicted) recruited in four centres each wore simultaneously three or four out of six commercially available monitors validated in chronic conditions for 14 consecutive days. A priori validity criteria were defined. These included the ability to explain total energy expenditure (TEE) variance through multiple regression analysis, using TEE as the dependent variable with total body water (TBW) plus several physical activity monitor outputs as independent variables; and correlation with activity energy expenditure (AEE) measured by DLW.

The Actigraph GT3X (Actigraph LLC, Pensacola, FL, USA), and DynaPort MoveMonitor (McRoberts BV, The Hague, the Netherlands) best explained the majority of the TEE variance not explained by TBW (53\% and 70\%, respectively) and showed the most significant correlations with AEE $(r=0.71, \mathrm{p}<0.001$ and $\mathrm{r}=0.70, \mathrm{p}<0.0001$, respectively).

The results of this study should guide users in choosing valid activity monitors for research or for clinical use in patients with chronic diseases such as COPD.

0

@ERSpublications

This study validates six activity monitors in the field against indirect calorimetry (DLW) in patients with COPD http://ow.ly/o9VIE

This article has supplementary material available from www.erj.ersjournals.com

Received: Aug 272012 | Accepted after revision: Dec 262012 | First published online: Feb 082013

Support statement: This work has been funded by the Innovative Medicine Initiative Joint Undertaking (IMI JU \# 115011). This work is supported by the NIHR Respiratory Biomedical Research Unit at Royal Brompton and Harefield NHS Foundation Trust and Imperial College London.

Conflict of interest: Disclosures can be found alongside the online version of this article at www.erj.ersjournals.com Copyright @ERS 2013 


\section{Introduction}

Chronic obstructive pulmonary disease (COPD) is a debilitating and progressive disease characterised by poorly reversible airflow limitation [1] and associated with several extrapulmonary effects, of which skeletal muscle dysfunction [2] contributes together with airflow limitation to limit the exercise capacity of these patients [3]. In association with psychological and behavioural aspects, the reduced exercise capacity contributes, in turn, to the reduced physical activity characteristic of patients with COPD [4, 5], a factor which limits social interaction and may threaten independence.

Inactivity is associated with outcomes relevant to healthcare providers, such as lung function deterioration [6], hospital admission [7] and death [8]. Enabling patients to become more physically active and less symptomatic is, therefore, an important patient-centred goal for pharmacological and nonpharmacological therapies in COPD. Therefore, accurate assessment of physical activity may provide a unique perspective on treatment effectiveness.

Physical activity is defined as any bodily movement produced by the contraction of skeletal muscle that increases energy expenditure above a basal level [9]. There is consensus that physical activity is best evaluated by direct measurement using physical activity monitors (PAMs) [10, 11] rather than questionnaires [12]. The number of PAMs is growing with improvements in technology.

However, assessing physical activity in patients with less and slower activity than healthy subjects is challenging [12]. The accuracy of PAMs to detect subtle changes in physical activity needs to be validated, and their acceptability to, and usability by, patients needs to be assessed.

The European Union/Innovative Medicine Initiative-funded PROactive project (www.proactivecopd.com) is developing a patient-reported outcome (PRO) tool directed at physical activity in COPD. As part of this process, a prior systematic review [13] identified six commercially available PAMs, which have previously been validated against indirect calorimetry in healthy adult populations and in some chronic conditions. These monitors were selected for further validation.

Our operational definition of validity was determined a priori at a consortium meeting in which stakeholders were represented. We considered that the activity measure should show a good correlation with more direct measures of energy expenditure. As a direct measure of energy expenditure we used the doubly labelled water (DLW) indirect calorimetry technique [14]. In addition, we considered a series of highly desirable criteria for an activity monitor to be a valid tool to assess physical activity in COPD patients, as follows. First, a monitor should be able to capture small changes in physical activity [15]. Such differences are observed during weekends in patients with COPD [4]. Secondly, PAMs should be user friendly in order to be accepted by the patient measured.

Therefore, to further assess the utility of PAMs for use in clinical trials and in the PRO being developed we conducted a multicentre evaluation of the six commercially available monitors.

\section{Methods}

Study group

80 patients with COPD (61 male) were recruited across four European centres (Athens, Greece; Leuven, Belgium; and Edinburgh and London, UK) (20 patients per centre) [16]. All had a diagnosis of COPD according to the Global Initiative for Chronic Obstructive Lung Disease [1]. They were clinically stable and free of exacerbations for 4 weeks prior to the study. Patients were excluded if they had comorbidities which would interfere with their movement, cognitive impairment, or if they were on variable doses of diuretics which would potentially interfere with the DLW method. The study was approved by the local ethics committees in each country as well as by the independent ethical board of the PROactive project, and written informed consent was obtained from the participants.

\section{Study design}

This was a multicentre cross-sectional validation study with 14 days of continuous assessment. The six monitors were randomly assigned to the different centres (three monitor types per centre). Patients were asked to wear simultaneously during wakefulness up to four PAMs (including the three monitors assigned to the centre) out of the six monitors selected for validation [13]: two uniaxial monitors (Lifecorder PLUS (Kenz Suzuken Co Ltd, Nagoya, Japan) and Actiwatch Spectrum (Philips Respironics, Bend, OR, USA)), three triaxial monitors (Actigraph GT3X (Actigraph LLC, Pensacola, FL, USA), DynaPort MoveMonitor (McRoberts BV, The Hague, the Netherlands) and RT3 (StayHealthy Inc., Monrovia, CA, USA)) and one multisensor monitor, a triaxial accelerometer with different sensors (SenseWear Armband; Bodymedia Inc., Pittsburgh, PA, USA) (table 1). 
TABLE 1 Details of type, location and available outputs for the six activity monitors

\begin{tabular}{|c|c|c|c|c|c|c|}
\hline Name; manufacturer (software) & Type & Subjects & Days & Location & $\begin{array}{l}\text { Measured } \\
\text { output }\end{array}$ & $\begin{array}{l}\text { Estimated } \\
\text { output }\end{array}$ \\
\hline $\begin{array}{l}\text { Lifecorder PLUS; Kenz Suzuken Co Ltd, } \\
\text { Nagoya, Japan (Physical Activity Analysis } \\
\text { Software) }\end{array}$ & $\begin{array}{l}\text { Uniaxial } \\
\text { accelerometer }\end{array}$ & 40 & 471 & Waist (left) & Activity score & TEE \\
\hline $\begin{array}{l}\text { Actiwatch Spectrum; Philips Respironics, } \\
\text { Bend, OR, USA (Respironics Actiware 5) }\end{array}$ & $\begin{array}{l}\text { Uniaxial } \\
\text { accelerometer }\end{array}$ & 40 & 453 & Wrist (left) & Activity counts & \\
\hline $\begin{array}{l}\text { Actigraph GT3X; Actigraph LLC, Pensacola, } \\
\text { FL, USA (Actilife 5) }\end{array}$ & $\begin{array}{l}\text { Triaxial } \\
\text { accelerometer }\end{array}$ & 39 & 463 & Waist (right) & $\begin{array}{l}\text { Activity time, } \\
\text { vector magnitude } \\
\text { units, steps }\end{array}$ & \\
\hline $\begin{array}{l}\text { DynaPort MoveMonitor; McRoberts BV, } \\
\text { the Hague, the Netherlands (www. } \\
\text { mcroberts.nl) }\end{array}$ & $\begin{array}{c}\text { Triaxial } \\
\text { accelerometer }\end{array}$ & 40 & 443 & $\begin{array}{l}\text { Waist (lower } \\
\text { back) }\end{array}$ & $\begin{array}{l}\text { Steps, movement } \\
\text { intensity, } \\
\text { different body } \\
\text { positions }\end{array}$ & TEE \\
\hline
\end{tabular}

Data are presented as n. TEE: total energy expenditure; AEE: activity energy expenditure.

Patients wore the activity monitors during wakefulness for 14 consecutive days, and were instructed not to wear them while bathing or swimming. Their outputs over this period were compared to a gold standard of indirect calorimetry as obtained from DLW (see later). The monitors provide measured outputs derived from accelerometer data and estimated energy expenditure outputs derived from these and other variables (i.e. anthropometric data of the patient) using prediction equations (table 1). We aimed at providing validity for the measured outputs of the devices rather than their prediction of energy expenditure, as this is highly dependent on reference equations (which are not available to users in some cases for commercial reasons and are not provided by all six monitors) to convert accelerometer data into energy equivalents.

At the end of the monitoring period, patients completed a usability questionnaire for each monitor, which assessed factors such as ease of use, comfort and obtrusiveness. The questionnaire is available in the online supplementary material.

The following a priori criteria for validation of the monitors were established: 1) significant relationship with DLW-derived active energy expenditure; 2) ability to capture day-to-day variability in physical activity levels throughout the period of assessment, since these are a recognised feature of COPD [15]; 3) ability to capture the lower levels of activity during weekends, characteristic of COPD patients [4]; and 4) acceptable patient-reported usability criteria.

Patients visited the centres on three occasions, with 1 week between visits.

\section{Assessments}

All patients underwent the following baseline assessments: anthropometric measurements, pulmonary function tests (spirometry, lung diffusion capacity and lung volumes), 6-min walking distance (6MWD), incremental cardiopulmonary exercise test and COPD-specific health-related quality-of-life questionnaires (St George's Respiratory Questionnaire, COPD assessment tool, modified Medical Research Council (mMRC) dyspnoea scale and usability questionnaire). Full details of the assessments are listed in the online supplementary material.

\section{DLW method}

The total energy expenditure (TEE) over the 14-day period of monitoring was measured using DLW, according to the Maastricht protocol [14]. On the morning of day 0, body weight was recorded, and after the collection of a background urine sample, subjects drank a weighed amount of DLW (enriched with ${ }^{2} \mathrm{H}$ and ${ }^{18} \mathrm{O}$, such that baseline levels of DLW (normally present in tap water and in urine) were increased by 
$100 \mathrm{ppm}$ for ${ }^{2} \mathrm{H}$ and $200 \mathrm{ppm}$ for ${ }^{18} \mathrm{O}$ ). Urine samples were collected in the afternoon of day 0 approximately $6 \mathrm{~h}$ post-ingestion and from the second voiding. Further samples were collected on the mornings of day 1, day 7 and day 14, and in the evening of day 6 and day 13. Patients were asked to note carefully the exact time of each urine sample. The DLW method provides an average value of TEE per day during the period of assessment.

Resting energy expenditure (REE) was measured using the ventilated hood method [17]. An open-circuit, computerised indirect calorimeter (Deltatrac; Datex Division, Helsinki, Finland) connected to a transparent hood system was used. Patients were instructed to fast overnight and measurements were taken first thing in the morning of the first day of the study period. The calorimeter was calibrated before each patient measurement using a span gas $\left(20 \% \mathrm{CO}_{2}\right.$ and $\left.1 \% \mathrm{O}_{2}\right)$, and to nitrogen for a zero calibration.

Patients were asked to lie still under the hood for 20-30 min until the readings of oxygen uptake $\left(V^{\prime} \mathrm{O}_{2}\right)$ and carbon dioxide production $\left(V^{\prime} \mathrm{CO}_{2}\right)$ had reached equilibrium. The mean of the following $10 \mathrm{~min}$ of readings were taken to calculate resting energy expenditure. A second measurement was taken during the second week of the study and a mean of the two values was used. The Weir equation [18] was used to calculate resting energy expenditure from the $V^{\prime} \mathrm{O}_{2}$ and $V^{\prime} \mathrm{CO}_{2}$ values obtained.

Active energy expenditure (AEE) was measured as $(0.9 \times \mathrm{TEE})$-REE, assuming diet-induced thermogenesis to be $10 \%$ of TEE [19].

Due to technical problems, some of the measurements of REE were not accurate (i.e. very low values of REE) and 11 patients have to be excluded from any analysis involving AEE. Three other patients were excluded due to technical problems with the DLW assessment.

Total body water (TBW) was calculated as the ${ }^{2} \mathrm{H}$ dilution space from the ${ }^{2} \mathrm{H}$ enrichment of the second voiding minus the ${ }^{2} \mathrm{H}$ concentration of the sample before dose administration [20]. The ${ }^{2} \mathrm{H}$ dilution space was divided by 1.04 to correct for isotope exchange with nonaqueous hydrogen of body solids [21].

\section{Statistical analysis}

Results are described as mean \pm SEM unless otherwise specified. A priori criteria for monitor validity were established before any analyses were undertaken. Being physical activity defined as "body movement incurring in energy consumption", we expected a significant correlation between the outputs of the monitors (based on accelerometer data to capture movement) with the energy consumed during the same period of assessment using DLW. The relationship of each activity monitor output (average of the whole period of assessment) with active energy expenditure (AEE) as assessed by DLW was tested by Pearson's correlation (see online supplementary figure S1 for Bland-Altman plot for those monitors giving an estimate of mean total daily energy expenditure).

A significant portion of the total energy consumed obtained with the DLW method would be related to the body composition of a particular subject. Therefore, we further explored the ability of the activity monitors to explain the variance of TEE not correlated with TBW (the portion of TEE related to movement). In order to explain the variability in TEE measured with the DLW method, a multiple stepwise regression analysis was performed with TEE as the dependent variable and DLW-measured TBW (a surrogate for metabolically active tissue and hence REE) and PAM outcomes as independent variables [22].

The ability of each PAM to capture variability in physical activity level was assessed by comparison of the coefficient of variation of monitor outcomes (one value per day of assessment) over the 14 days of assessment using one-way ANOVA. The ability to capture lower levels of activity present at weekends, characteristic of COPD patients [4], was tested by t-test to compare weekday versus weekend activity for each monitor (average of each period weekday/weekend). Relationship with parameters of exercise (6MWD, peak $V^{\prime} \mathrm{O}_{2}$ ) was tested using Pearson's correlation.

Acceptable patient-reported usability was based on the questionnaire results. In order to assess the repeatability of physical activity outcomes assessed by PAMs, we calculated the differences between outputs obtained in the two consecutive weeks of assessment. We expected $95 \%$ of the differences between week 1 and week 2 to be less than two standard deviations (of the difference) [23]. A t-test was also used to compare PAM outputs between the first and second week of assessment. A comparison between the variability (standard deviation) for a representative measured variable for each of the six PAMs comparing the first week of assessment to the whole period of 14 days ( 2 weeks) was performed to evaluate whether 2 weeks of assessment add power (reduction of the standard deviation) to the physical activity evaluation.

The level of significance for all comparisons was set at $\mathrm{p}<0.05$. Data were analysed using the statistical package SAS version 9.2 (SAS Institute Inc., Cary, NC, USA). 


\section{Results}

Patients had at least two weekends and six weekdays of data for each activity monitor. The first and last day were excluded to eliminate bias (as these were incomplete days and included visits to the study centres). Although subjects wore the monitors for 14 days, in some cases technical problems, or insufficient hours of data collection, resulted in incomplete data collection (i.e. $<10 \mathrm{~h}$ of data during daytime hours per day). Out of all possible days of assessment, the proportion of days with $>10 \mathrm{~h}$ of assessment included in the validation analysis ranged from $79 \%$ to $91 \%$ for all six monitors (see the online supplementary material for details).

No patient had to be entirely excluded from the analyses. However, incomplete days of monitoring were excluded.

Anthropometric characteristics and pulmonary function data of patients are listed in table 2. The patients had a wide spectrum of disease severity in terms of lung function (forced expiratory volume in $1 \mathrm{~s}$ (FEV1) from $16 \%$ predicted to $96 \%$ predicted and Global Initiative for Chronic Obstructive Lung Disease (GOLD) stage I $n=10$, stage II $n=43$, stage III $n=20$ and stage IV $n=7$ ) and in terms of symptoms (mMRC score 1 $n=12,2 n=33,3 n=26$ and $4 n=9$ ). No differences in total or activity-related energy expenditure measured with the DLW method were identified between patients wearing different monitors. Moreover, no differences were observed in body composition, lung function, dyspnoea score, BODE (body mass index, airflow obstruction, dyspnoea and exercise capacity) index and health-related quality of life (see online supplementary material).

\section{PAM validation against DLW}

Measured (instead of estimated) activity monitor outputs that showed the most significant correlations with AEE measured by the DLW method are shown in figure 1 (for a broader list of variables see the online supplementary material). All the monitors showed statistically significant correlations with AEE. The better the correlation, the better the ability of a monitor to identify a patient with high AEE as being a more active patient and a patient with a low AEE as being a less active patient.

As expected, most of the total energy expenditure variance was explained by TBW (a surrogate of REE) (fig. 2). Although all monitors significantly explained part of the residual variance with some of the variables remaining in the model $(\mathrm{p}<0.05)$, the DynaPort MoveMonitor $\left(\mathrm{r}^{2}=0.30\right)$ (average movement intensity + walking time + walking movement intensity), and the Actigraph GT3X $\left(\mathrm{r}^{2}=0.24\right)$ (activity time) were the two monitors with the largest variance explained by the monitor in the model, explaining $70 \%$ and $53 \%$ of the TEE variance not correlated with TBW respectively. The remaining monitors explained a lower portion of the TEE variance not explained by TBW (Actiwatch Spectrum (activity count; $\mathrm{r}^{2}=0.18$ ); SenseWear (average metabolic equivalents of task + steps; $\mathrm{r}^{2}=0.08$ ); Lifecorder PLUS (activity score; $\left.\mathrm{r}^{2}=0.10\right)$; and RT3 $\left(\mathrm{TEE} ; \mathrm{r}^{2}=0.07\right)$ ). Because three different variables corresponding to the DynaPort MoveMonitor contribute to explain the TEE variance not explained by TBW, we have conducted the analysis with the variable with the highest contribution (average movement intensity). Considering this variable alone $\left(r^{2}=0.23\right)$ the percentage of the possible explained variance drops from $70 \%$ to $54 \%$. The GT3X and the DynaPort remain the monitors with the largest variance explained by the monitor in the model.

\section{Ability of PAMs to capture variability and differences in physical activity between weekends and weekdays}

The ability of activity monitors to capture variability, assessed as the coefficient of variation for the different monitor's outputs is shown in figure 3a. All monitors were able to capture variability in physical activity levels across the different days throughout the period of assessment. The ability of the monitors to detect changes in physical activity levels was also assessed as the differences between weekend and weekday physical activity levels (fig. 3b). With exception of the RT3, all monitors were capable of capturing differences between weekends and weekdays.

\section{Usability of activity monitors}

Patient adherence with the use of the monitors (despite using three or four simultaneously) was good throughout the period of assessment, as reflected by the compliance range from $79 \%$ to $91 \%$ (online supplementary table S1) and by the usability questionnaire. Figure 4 illustrates the scores from the questionnaire used to assess usability. Most patients declare willingness to wear most devices for more than a week, and the devices were generally not perceived as intrusive. Patients also provided an overall score on the monitors. In general the scores were good, ranging from 75 to 91 on a scale from 0 (worst score) to 100 (best score). 


\section{TABLE 2 Characteristics of the study group}

Male/female
Age years
BMI $\mathrm{kg} \cdot \mathrm{m}^{-2}$
mMRC
FEV1 L
FEV $1 \%$ pred
FVC L
FVC \% pred
FEV $1 /$ FVC
BODE index
6MWD m
Wpeak W
$V^{\prime} 0_{2}$ peak $\mathrm{mL} \cdot \mathrm{min}^{-1} \cdot \mathrm{kg}^{-1}$
SGRQ total score
CAT
AEE $\mathrm{kCal} \cdot \mathrm{kg}^{-1}$
TBW L

$$
\begin{gathered}
61 / 19 \\
68 \pm 6.2 \\
26.5 \pm 4.7 \\
2.4 \pm 0.9 \\
1.5 \pm 0.6 \\
57 \pm 19.1 \\
3.2 \pm 0.8 \\
95 \pm 20.1 \\
0.5 \pm 0.1 \\
5.7 \pm 1.7 \\
435 \pm 118.1 \\
81 \pm 40.1 \\
17.1 \pm 5.9 \\
42 \pm 20.1 \\
16 \pm 7.5 \\
11.4 \pm 4.5 \\
35.6 \pm 6.6
\end{gathered}
$$

Data are presented as $\mathrm{n}$ or mean \pm SD. BMI: body mass index; mMRC: modified Medical Research Council dyspnoea score; FEV1: forced expiratory volume in $1 \mathrm{~s} ; \%$ pred: \% predicted; FVC: forced vital capacity; BODE: BMI, airflow obstruction, dyspnoea and exercise capacity; 6MWD: 6-min walk distance; Wpeak: power achieved during the incremental exercise test; $V^{\prime} \mathrm{O}_{2}$ peak: peak oxygen uptake achieved during the incremental exercise test; SGRQ: St George's Respiratory Questionnaire; CAT: chronic obstructive pulmonary disease assessment test; AEE: activity energy expenditure; TBW: total body water.

\section{Discussion}

The main findings of this multicentre study were that the output from all monitors demonstrated a significant relationship with AEE derived from DLW measurements in line with those typically observed in field validation studies [13, 24-28]. The DynaPort MoveMonitor and the Actigraph GT3X best explained the activity related energy from TEE not related to TBW. Few studies have individually validated these PAMs (or their older versions) in the field in chronic disease populations [13]. This is the first multicentre trial where several activity monitors were validated head-to-head against directly measured energy expenditure at different stages of COPD.

The validity of the PAMs against energy expenditure measured by the DLW method in the present study was assessed using correlation analysis, rather than a measure of agreement (e.g. Bland-Altman analysis) (see online supplementary material). There are two main arguments in support of this approach. First, we were more interested in providing validity for the measured outputs of the devices rather than their prediction of energy expenditure, as this is highly dependent on reference equations to convert accelerometry into energy equivalents (which are not available to users in some cases for commercial reasons). In addition, only three out of the six monitors explored provide estimated values of total energy expenditure. Physical activity is defined as any bodily movement produced by skeletal muscle resulting in energy consumption. It is therefore reasonable to expect and investigate a relation between movement measured by accelerometers and energy expenditure assessed with indirect calorimetry (DLW) throughout the same period as proof of validity. Second, energy expenditure is driven to a large extent by subject specific characteristics including body weight, age, height and specific factors such as mechanical efficiency. It is not feasible to take mechanical efficiency into account in predicting energy expenditure, especially in patients with COPD.

COPD patients have a poor mechanical efficiency yielding larger energy expenditure compared to healthy subjects [29-32]. Consequently, AEE remains relatively well preserved whereas it is well recognised that patients are moving less [32]. It cannot be expected that an activity monitor would be able to take the individual's mechanical efficiency into account and provide very accurate estimates of AEE. Hence, we suggest that activity monitors should be used to assess the activities (i.e. movements) of patients in terms of amount and/or intensity of activity and that greater weight should be given to direct monitor outputs (steps, activity count, vector magnitude units, etc.). We acknowledge that the derivation of energy expenditure is difficult by default and probably inaccurate when based on acceleration signals only, but consider that this does not render activity monitors invalid for the assessment of bodily movement and, perhaps even more importantly, intervention-associated changes. Therefore, comparing the raw data from 
the monitors with the AEE (derived from the DLW method) by using correlation analysis was judged as the appropriate statistical method [33].

All monitors were able to capture variability in activity levels across the different days throughout the period of assessment. Moreover, with the exception of the RT3 device, monitors were able to capture differences between weekends and weekdays.

Another interesting finding was the good correlation between activity monitor variables and physiological variables reflecting exercise capacity (see online supplementary material). It is to be expected that patients with higher exercise capacity perform higher levels of activity and this should be captured by the monitors. The Lifecorder PLUS monitor showed weak correlations with exercise capacity variables, whereas the Actigraph GT3X showed the best correlations with all outcomes of exercise capacity. For the DynaPort MoveMonitor and the SenseWear Armband the correlations were in line with those reported previously with older models of these monitors $[4,5,34]$.

Besides the concepts of validity and reliability, other factors such as size and scope of the study, usability and cost of the monitor must be taken into consideration when selecting a monitor for clinical trials. Patients participating in the present study were asked to rate their perception of wearing the devices using a 12-item
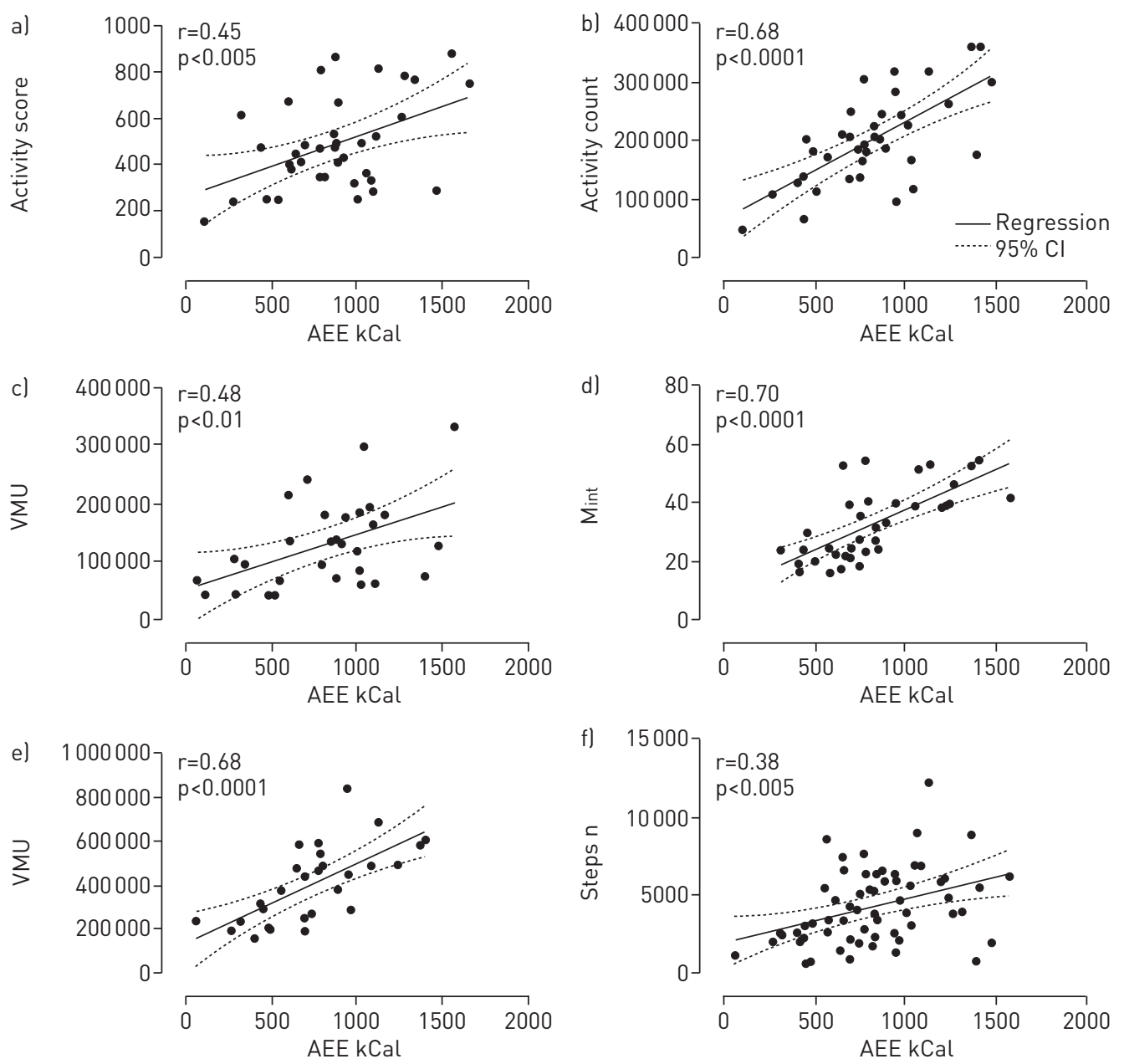

FIGURE 1 Correlations of individual data of physical activity measured outcomes with active energy expenditure (AEE) measured with doubly labelled water for the six monitors studied: a) Lifecorder PLUS (Kenz Suzuken Co Ltd, Nagoya, Japan); b) Actiwatch Spectrum (Philips Respironics, Bend, OR, USA); c) RT3 (StayHealthy Inc., Monrovia, CA, USA); d) DynaPort MoveMonitor (McRoberts BV, the Hague, the Netherlands); e) Actigraph GT3X (Actigraph LLC, Pensacola, FL, USA); f) SenseWear Armband (Bodymedia Inc., Pittsburgh, PA, USA). VMU: vector magnitude units; Mint: movement intensity. 


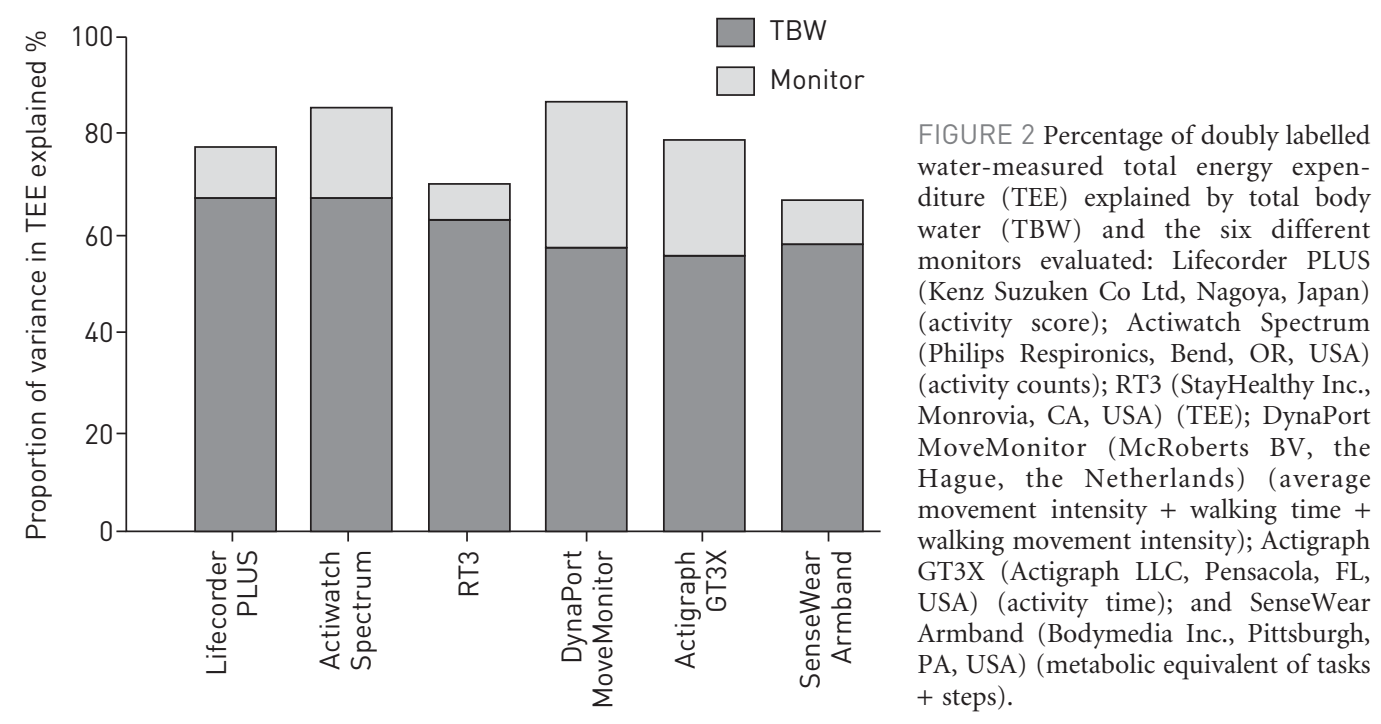

questionnaire designed for this purpose. Most patients did not mind wearing most devices for more than a week, and the devices were generally not perceived as intrusive (fig. 4).

Our analysis comparing the first and second week of activity assessment showed repeatable data in 2 consecutive weeks in this population of COPD patients (see online supplementary material). Moreover, adding a second week of measurements did not significantly change the variability, whether it was based on 1 or 2 weeks of measurement, indicating that there would be little or no advantage, in terms of a reduction in the sample size required, of extending the period of assessment from 1 week to 2 weeks.

\section{Limitations of the study}

Although patients of all GOLD stages were studied, there was not an even distribution. However, there was a wide range in activity levels (as energy consumed during activities (AEE) assessed with DLW) (55.4-1581.2 kCal) and 6MWD (149-675 m), so we consider this study group to be representative of the spectrum of activity levels in COPD, and of patients typically entering clinical trials.

None of the patients wore all six monitors simultaneously, which precluded direct comparison of all six monitors in the same group of patients. This does not compromise the results of the present study, since the main results are based in the individual comparison of each monitor with DLW-derived variables. Moreover, wearing all monitors would have been onerous and could have introduced other errors such as poorer compliance. We felt that each patient wearing up to four monitors was a good compromise. Another potential limitation of the study relates to the exclusion of 11 patients for the calculation of AEE due to

a)

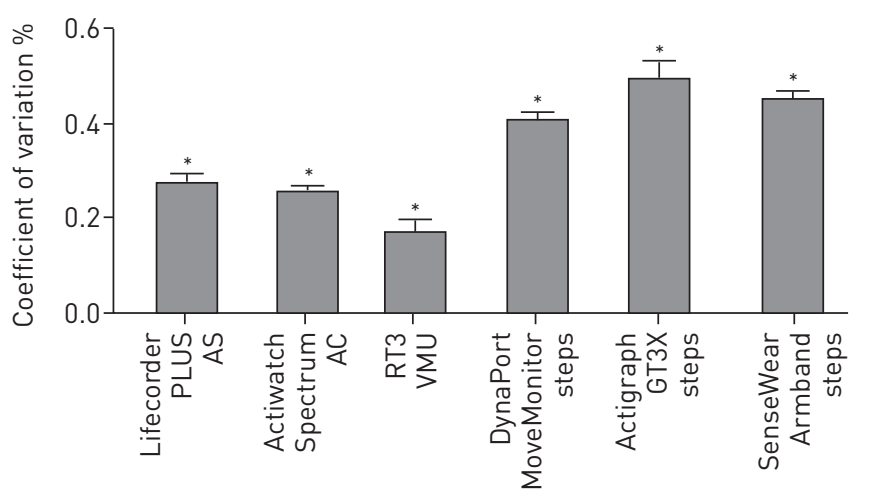

b)

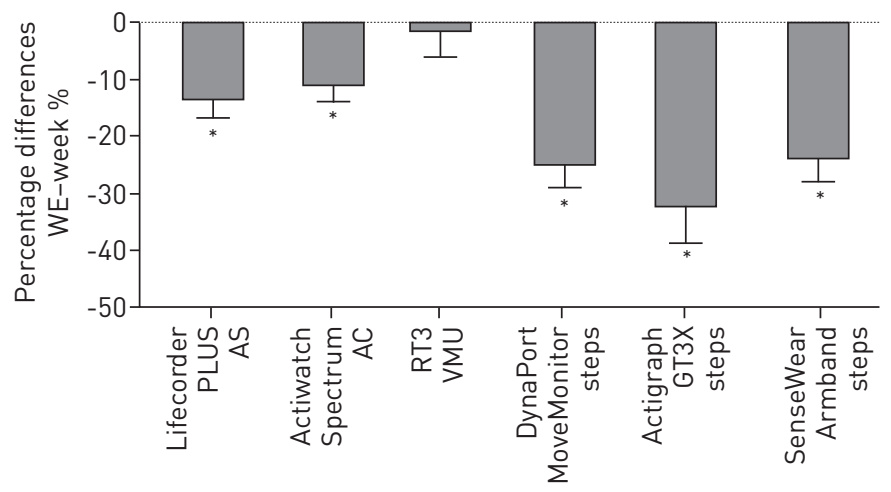

FIGURE 3 Variability of physical activity outcomes for the six activity monitors. a) Ability of all monitors to capture variability of the data throughout the 14-day period of assessment; b) ability of monitors to capture changes in physical activity between weekends (WE) and weekdays (week). Error bars indicate SEM. VMU: vector magnitude units; AS: activity score; AC: activity count. ${ }^{*}: \mathrm{p}<0.05$. 

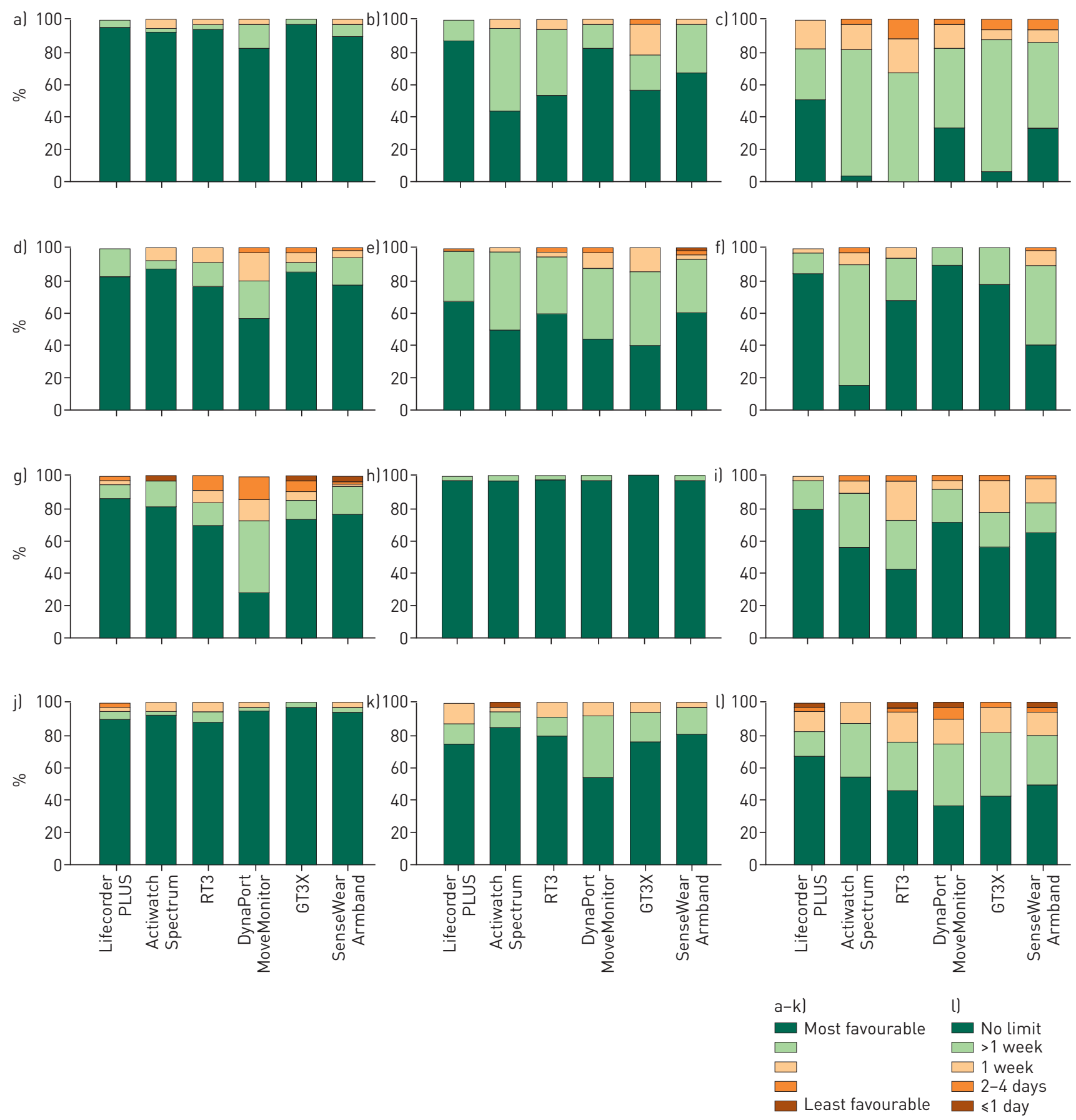

FIGURE 4 Usability of physical activity monitors as assessed directly by 71 patients who completed the usability questionnaire. The questions were as follows. a) "I experienced technical problems"; b) "The instructions on how to use the monitor were clear"; c) "The monitors were bulky/heavy"; d) "The monitor interfered with my normal activities"; e) "Using the monitor on a daily basis was easy"; f) "The monitor bothered me in bed"; g) "I felt comfortable wearing the monitor"; h) "How much trouble did you have getting started with the monitor?"; i) "I felt my privacy was invaded by the monitor"; j) "I felt embarrassed wearing the monitor"; k) "The monitors were easy to put on/take off"; and l) "How long would you be willing to wear this particular monitor?".

technical problems in the assessment of REE. We could have calculated REE from Harris-Benedict equations but we didn't feel that this approach was more appropriate than using our own data on REE, due to known elevated REE in patients with COPD. However, we have run the validation analysis with AEE data calculated with Harris-Benedict equations, which lead us to similar conclusions as the present analysis.

In summary, all tested monitors showed good correlations with DLW-measured AEE. The best correlations corresponded to two of the triaxial monitors tested (the DynaPort MoveMonitor and the Actigraph GT3X). These monitors were also the best able to explain variability in TEE-DLW associated with physical activity, 
and were therefore most representative of what patients were actually doing. These results complement the results for the previously published laboratory validation study of the same six monitors [33], and taken together may allow users to choose the most appropriate activity monitors and end-points for research or for clinical use in patients with COPD. Our data also provide a consensus benchmark for future monitors.

\section{Acknowledgements}

The authors would like to acknowledge the members of the PROactive consortium for their outstanding contribution to this work.

PROactive consortium members are as follows. C. Brindicci and T. Higenbottam: Chiesi Farmaceutici S.A., Parma, Italy; T. Troosters and F. Dobbels: Katholieke Universiteit Leuven, Leuven, Belgium; M.X. Tabberer: GlaxoSmithKline, London, UK; R.A. Rabinovich and W. McNee: University of Edinburgh, Edinburgh, UK; I. Vogiatzis: Thorax Research Foundation, Athens, Greece; M. Polkey and N. Hopkinson: Royal Brompton and Harefield NHS Foundation Trust, London, UK; J. Garcia-Aymerich: Municipal Institute of Medical Research, Barcelona, Spain; M. Puhan and A. Frei: Universität Zürich, Zürich, Switzerland; T. van der Molen and C. De Jong: University Medical Centre, Groningen, the Netherlands; P. de Boer: Netherlands Asthma Foundation, Leusden, the Netherlands; I. Jarrod: British Lung Foundation, London, UK; P. McBride: Choice Healthcare Solutions, London, UK; N. Kamel: European Respiratory Society, Brussels, Belgium; K. Rudell and F.J. Wilson: Pfizer, Sandwich, UK; N. Ivanoff: Almirall, Barcelona, Spain; K. Kulich and A. Glendenning: Novartis, Basel, Switzerland; N.X. Karlsson and S. Corriol-Rohou: AstraZeneca AB, Mölndal, Sweden; E. Nikai: UCB, Brussels, Belgium; D. Erzen: Boehringer Ingelheim, Ingelheim, Germany.

\section{References}

1 Rodriguez-Roisin R, Anzueto A, Bourbeau J, et al. Global Strategy for the Diagnosis, Management, and Prevention of Chronic Obstructive Pulmonary Disease. NHLBI/WHO Global Initiative for Chronic Obstructive Lung Disease (GOLD) Workshop summary. GOLD, 2010. Available from: www.goldcopd.com

2 Agustí AG, Noguera A, Sauleda J, et al. Systemic effects of chronic obstructive pulmonary disease. Eur Respir J 2003; 21: $347-360$.

3 Saey D, Debigare R, LeBlanc P, et al. Contractile leg fatigue after cycle exercise: a factor limiting exercise in patients with chronic obstructive pulmonary disease. Am J Respir Crit Care Med 2003; 168: 425-430.

Watz H, Waschki B, Meyer T, et al. Physical activity in patients with COPD. Eur Respir J 2009; 33: 262-272.

Pitta F, Troosters T, Spruit MA, et al. Characteristics of physical activities in daily life in chronic obstructive pulmonary disease. Am J Respir Crit Care Med 2005; 171: 972-977.

6 Garcia-Aymerich J, Lange P, Benet M, et al. Regular physical activity modifies smoking-related lung function decline and reduces risk of chronic obstructive pulmonary disease: a population-based cohort study. Am J Respir Crit Care Med 2007; 175: 458-463.

7 Garcia-Aymerich J, Lange P, Benet M, et al. Regular physical activity reduces hospital admission and mortality in chronic obstructive pulmonary disease: a population based cohort study. Thorax 2006; 61: 772-778.

8 Waschki B, Kirsten A, Holz O, et al. Physical activity is the strongest predictor of all-cause mortality in patients with COPD: a prospective cohort study. Chest 2011; 140: 331-342.

9 Caspersen CJ, Powell KE, Christenson GM. Physical activity, exercise, and physical fitness: definitions and distinctions for health-related research. Public Health Rep 1985; 100: 126-131.

10 Pitta F, Troosters T, Probst VS, et al. Physical activity and hospitalization for exacerbation of COPD. Chest 2006; 129: $536-544$.

11 Pitta F, Troosters T, Spruit MA, et al. Activity monitoring for assessment of physical activities in daily life in patients with chronic obstructive pulmonary disease. Arch Phys Med Rehabil 2005; 86: 1979-1985.

12 Cyarto EV, Myers AM, Tudor-Locke C. Pedometer accuracy in nursing home and community-dwelling older adults. Med Sci Sports Exerc 2004; 36: 205-209.

13 Van Remoortel H, Giavedoni S, Raste Y, et al. Validity of activity monitors in health and chronic disease: a systematic review. Int J Behav Nutr Phys Act 2012; 9: 84.

14 Westerterp KR, Wouters L, van Marken Lichtenbelt WD. The Maastricht protocol for the measurement of body composition and energy expenditure with labeled water. Obes Res 1995; 3: Suppl. 1, 49-57.

15 Hecht A, Ma S, Porszasz J, et al. Methodology for using long-term accelerometry monitoring to describe daily activity patterns in COPD. COPD 2009; 6: 121-129.

16 Rabe KF, Hurd S, Anzueto A, et al. Global strategy for the diagnosis, management, and prevention of chronic obstructive pulmonary disease: GOLD executive summary. Am J Respir Crit Care Med 2007; 176: 532-555.

17 Maffeis C, Schutz Y, Micciolo R, et al. Resting metabolic rate in six- to ten-year-old obese and nonobese children. J Pediatr 1993; 122: 556-562.

18 Weir JB. New methods for calculating metabolic rate with special reference to protein metabolism. J Physiol 1949; 109: 1-9.

19 Westerterp KR. Diet induced thermogenesis. Nutr Metab (Lond) 2004; 1: 5.

20 van Marken Lichtenbelt WD, Westerterp KR, Wouters L. Deuterium dilution as a method for determining total body water: effect of test protocol and sampling time. Br J Nutr 1994; 72: 491-497.

21 Schoeller DA, van Santen E, Peterson DW, et al. Total body water measurement in humans with ${ }^{18} \mathrm{O}$ and ${ }^{2} \mathrm{H}$ labeled water. Am J Clin Nutr 1980; 33: 2686-2693.

22 Bonomi AG, Plasqui G, Goris AH, et al. Estimation of free-living energy expenditure using a novel activity monitor designed to minimize obtrusiveness. Obesity (Silver Spring) 2010; 18: 1845-1851.

23 British Standards Institution. Precision of Test Methods 1: Guide for the Determination and Reproducibility for a Standard Test Method (BS 597, Part 1). London, BSI, 1975.

24 Adams SA, Matthews CE, Ebbeling CB, et al. The effect of social desirability and social approval on self-reports of physical activity. Am J Epidemiol 2005; 161: 389-398.

25 Assah FK, Ekelund U, Brage S, et al. Predicting physical activity energy expenditure using accelerometry in adults from sub-Sahara Africa. Obesity (Silver Spring) 2009; 17: 1588-1595. 

conditions. Med Sci Sports Exerc 2001; 33: 1233-1240.

27 Jacobi D, Perrin AE, Grosman N, et al. Physical activity-related energy expenditure with the RT3 and TriTrac accelerometers in overweight adults. Obesity (Silver Spring) 2007; 15: 950-956.

28 Mackey DC, Manini TM, Schoeller DA, et al. Validation of an armband to measure daily energy expenditure in older adults. J Gerontol A Biol Sci Med Sci 2011; 66: 1108-1113.

29 Sala E, Roca J, Marrades RM, et al. Effects of endurance training on skeletal muscle bioenergetics in chronic obstructive pulmonary disease. Am J Respir Crit Care Med 1999; 159: 1726-1734.

30 Richardson RS, Sheldon J, Poole DC, et al. Evidence of skeletal muscle metabolic reserve during whole body exercise in patients with chronic obstructive pulmonary disease. Am J Respir Crit Care Med 1999; 159: 881-885.

31 Franssen FM, Wouters EF, Baarends EM, et al. Arm mechanical efficiency and arm exercise capacity are relatively preserved in chronic obstructive pulmonary disease. Med Sci Sports Exerc 2002; 34: 1570-1576.

32 Baarends EM, Schols AM, Pannemans DL, et al. Total free living energy expenditure in patients with severe chronic obstructive pulmonary disease. Am J Respir Crit Care Med 1997; 155: 549-554.

33 Van Remoortel H, Raste Y, Louvaris Z, et al. Validity of six activity monitors in chronic obstructive pulmonary disease: a comparison with indirect calorimetry. PloS One 2012; 7: e39198.

34 Troosters T, Sciurba F, Battaglia S, et al. Physical inactivity in patients with COPD, a controlled multi-center pilotstudy. Respir Med 2010; 104: 1005-1011. 